\title{
Crystal Engineering of Polymeric Structures with Dative Bo- ron-Nitrogen Bonds: Design Criteria and Limitations
}

\author{
Nicolas Luisier, ${ }^{a}$ Kevin Bally, ${ }^{a}$ Rosario Scopelliti, ${ }^{a}$ Farzaneh T. Fadaei, ${ }^{a}$ Kurt Schenk, Philip Patti-

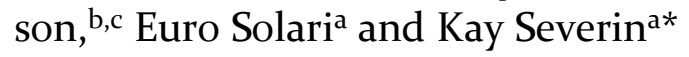 \\ ${ }^{a}$ Institute of Chemical Sciences and Engineering, École Polytechnique Fédérale de Lausanne (EPFL), CH-1015 Lau- \\ sanne, Switzerland \\ b Institute of Physics, École Polytechnique Fédérale de Lausanne (EPFL), CH-1015 Lausanne, Switzerland \\ c Swiss-Norwegian Beamlines at ESRF, 6 rue Jules Horowitz, 38043 Grenoble Cedex, France
}

\begin{abstract}
A data base analysis of known adducts between pyridyl donor ligands and Lewis-acidic boronate esters revealed a preference for a particular conformation at the boron centers. Based on this analysis, we designed two tritopic pyridyl ligands, which were expected to form polymeric structures upon combination with ditopic bis(benzenedioxaborole) Lewis acids. Instead of two-dimensional networks, we observed the formation of chains of macrocycles for different ester/Ndonor combinations. This kind of structural motif is unprecedented for polymeric B-N adducts. The predictability of the chemistry was compromised by the low strength of the dative B-N bond, which resulted in incomplete adduct formation and/or co-crystallization of starting materials.
\end{abstract}

\section{Introduction}

Boronate esters derived from condensation of arylboronic acids and catechols are Lewis acidic compounds, which can form adducts with $\mathrm{N}$-donor ligands such as pyridine. Adduct formation is accompanied by a distinct change of geometry of the boron center from trigonal planar to approximately tetrahedral (Scheme 1a). ${ }^{1}$ The utilization of polytopic boronate esters and polytopic N-donor ligands allows building more complex molecular architectures. ${ }^{2-12}$ For example, we were able to prepare cage-like structures by combining ditopic boronate esters and planar, rigid tripyridyl ligands (Scheme $1 b$ ). ${ }^{2}$ The inverse approach was followed by the group of Beuerle. They have prepared cages by linking triboronate esters with ditopic 1,4-diazabicyclo[2,2,2] octane (Scheme 1c). ${ }^{3}$ Instead of linking two different molecular building blocks via dative B-N bonds, it is also possible to employ boronate esters which feature N-donor ligands as side chains. Self-aggregation can then lead to the formation of molecularly defined and polymeric supramolecular structures. ${ }^{13-20}$

From a materials science perspective, the formation of crystalline networks is of special interest. In 2011, we reported first examples of two-dimensional networks based on dative B-N bonds. 7 These structures were obtained by combining triboronate esters with dipyridyl ligands of different lengths (Scheme 1d). The crystalline networks contain large voids, which are filled by disordered solvent molecules. Removal of the solvent lead to a collapse of the networks. However, it is conceivable that materials with permanent porosity can be prepared by employing stronger dative $\mathrm{B}-\mathrm{N}$ bonds (e.g. via tuning of electronic effects)..$^{20}$ a)

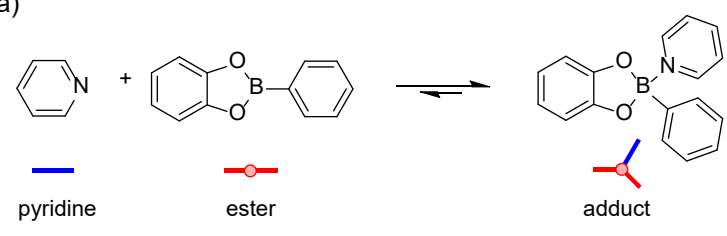

b)

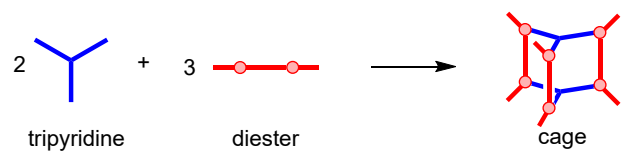

c)

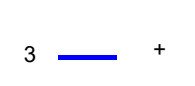

DABCO
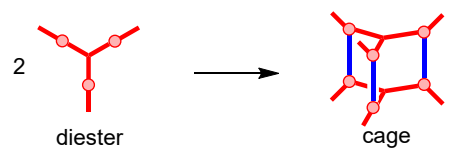

d)

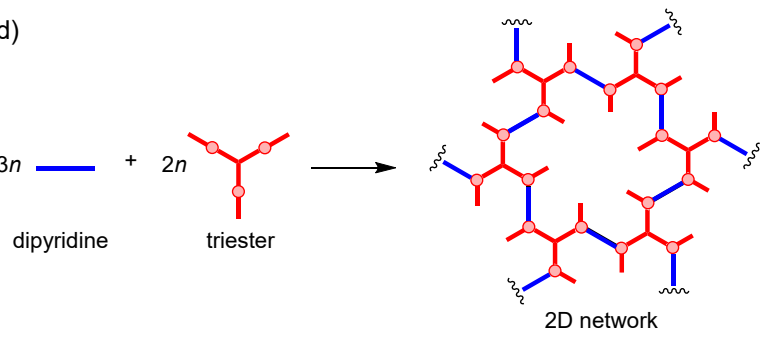

Scheme 1 The aggregation of N-donor ligands and boronate esters via dative $\mathrm{B}-\mathrm{N}$ bonds can be used to prepare molecular cages and crystalline, two-dimensional networks.

As depicted in Scheme 1, very similar strategies have been used to prepare molecular cages on one hand, and two-dimensional network structures on the other hand. In 
order to understand the factors which govern the self-assembly process, we have performed a geometrical analysis of crystalline compounds featuring pyridyl-boronate ester adducts. The analysis revealed a preference for a particular conformation at the boron centers. Based on this knowledge, we have identified two tripyridyl ligands, which were expected to form polymeric structures as opposed to molecularly defined nanostructures. The results of this study are summarized below.

\section{Results and Discussion}

Using the Cambridge Crystallographic Data Center (CCDC), we have extracted structural data of compounds which contain the motif shown in Figure 1, i.e. a pyridyl ligand bound to a boronate ester derived from a catechol and an arylboronic acid. Molecular as well as polymeric structures were taken into account, and all kinds of substituents at the rings were considered. For each symmetrically independent boron atom of these structures, we calculated the angles between the planes defined by the three aromatic rings (planes $\mathbf{b}, \mathbf{c}$, and $\mathbf{d}$ ) with respect to the reference plane defined by the N-B-C atoms (plane a, red). The exact values are given in the supporting information (Table $\mathrm{S}_{1}$ ) and a graphic representation is depicted in Figure 1.

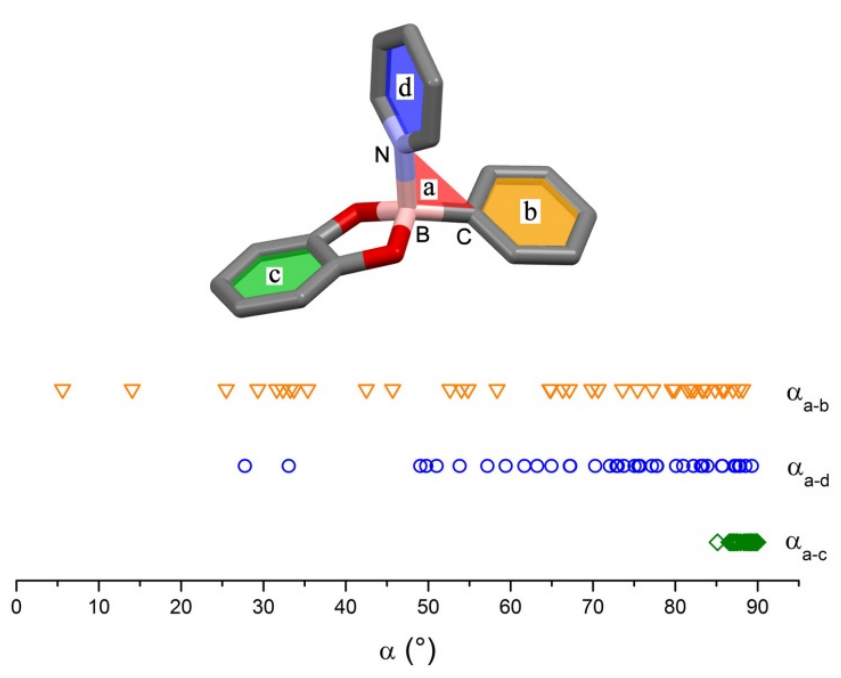

Figure 1 Angles between the planes defined by the aromatic rings of the arylboronate ester (orange, b), the pyridyl ligand (blue, d) or the catecholate (green, c) with respect to the reference plane defined by the three adjacent N-B-C atoms (red, a).

The catecholate plane $\mathbf{c}$ is always orthogonal to the reference plane a. This finding is not unexpected, given the fixation of plane c via two oxygen atoms to the boron center. More interestingly, we also observed a pronounced preference for a nearly orthogonal arrangement of the planes $\mathbf{d}$ and $\mathbf{a}$, with two thirds of the angles having values between 70 and $90^{\circ}$. For the planes $\mathbf{a}$ and $\mathbf{b}$, however, a more statistical distribution was detected.
The preferential orientation of the planes $\mathbf{d}$ and a provides an interesting opportunity with respect to the structural design of pyridyl-boronate ester adducts. Let's consider tripyridyl ligands with $D_{3 h}$ symmetry. If the planes defined by the pyridyl rings are co-planar with the $\sigma_{h}$ plane, then the boronate esters are preferentially oriented either up or down. This kind of orientation should favor the formation of cage-like structures if linear diboronate esters are employed as reactions partners (Scheme 2a). On the other hand, if the planes defined by the pyridyl rings are orthogonal to the $\sigma_{h}$ plane, then the boronate esters are preferentially oriented side-ways. Such an arrangement would favor the formation of polymeric structures (Scheme 2b).

a)
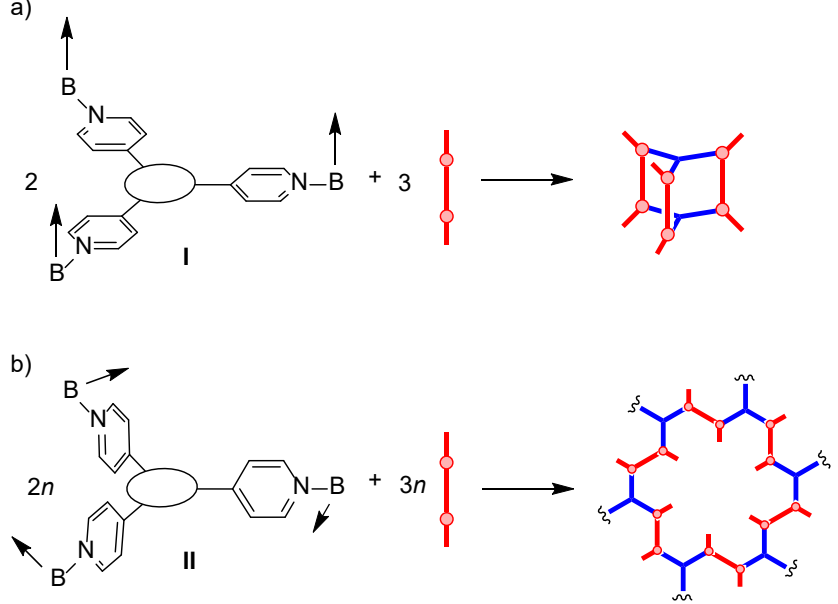

Scheme 2 Tripyridyl ligands and diboronate esters (red) can aggregate via dative $\mathrm{B}-\mathrm{N}$ bonds to give molecularly defined cages or polymers. 'Flat' ligands of type I should favor the formation of cages, whereas ligands with the conformation II should favor the formation of polymers.
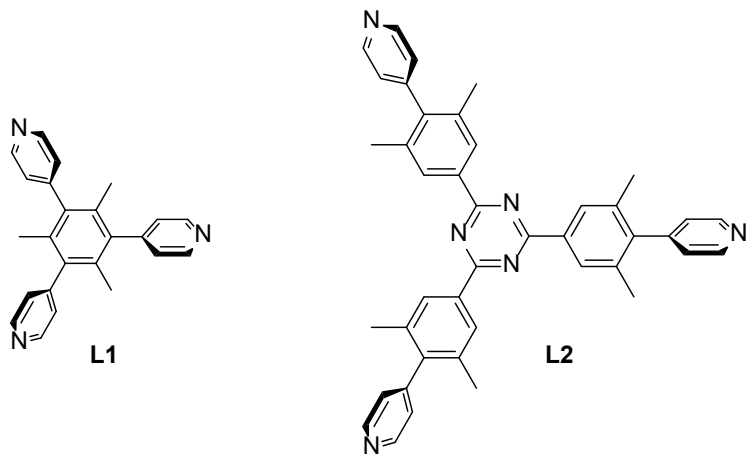

Figure 2 Molecular structures of the tripyridyl ligands L1 and L2.

In view of the arguments outlined above, we have synthesized the tripyridyl ligands L1 and L2 (Figure 2). Both ligands were expected to display conformation II, because the presence of methyl substituents should make an orthogonal arrangement of the pyridyl ring and the adjacent arene ring energetically more favorable. The synthesis of $\mathbf{L 1}$ was achieved by a triple Suzuki cross-coupling reaction of tribromomesitylene with 4-pyridylboronic acid. Ligand L2 
was obtained in 5 steps from commercially available 2,5 dibromo-m-xylene (for details see SI).

To corroborate our assumptions about the geometric predisposition of these ligands and their adducts, we prepared a simple adduct between L1 and the monotopic boronate ester E1 (Scheme 3). The molecular structure of the adduct $\mathbf{L r}(\mathbf{E} \mathbf{1})_{3}(\mathbf{A r})$ in the crystal confirmed that the pyridyl rings of $\mathbf{L} \mathbf{1}$ are indeed oriented orthogonal to the plane defined by the central mesityl ring (Figure 3). As predicted, the boronate esters are oriented side-ways, with the orientation being defined by the B-C bond axis. For reactions with ditopic, linear diboronate esters, such an arrangement should lead to the formation of polymeric structures.

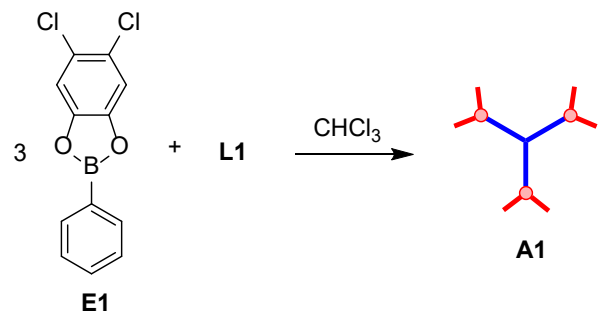

Scheme 3 Synthesis of the triple adduct A1.

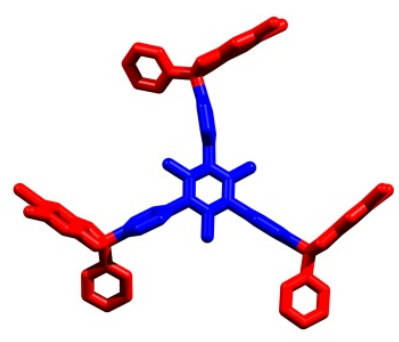

Figure 3 Molecular structure of the triple adduct A1 in the crystal. Color coding: blue: L1, red: E1. Hydrogen atoms and solvent molecules have been omitted for clarity.

Next, we combined the tripyridyl ligands L1 or L2 with different diboronate esters $\left(\mathbf{E}_{2}-\mathbf{E}_{5}\right)$. In order to obtain crystalline materials, we heated solutions of the respective mixtures until all starting compounds had dissolved (typically $>105^{\circ} \mathrm{C}$ ). Apolar organic solvents with a high boiling point such as toluene, 1,2-dichlorobenzene, or 1,2,4-trichlorobenzene were employed. The mixtures were then allowed to cool down in a controlled fashion with a temperature gradient of $-1{ }^{\circ} \mathrm{C} / \mathrm{h}$. For this purpose, we have used a programmable Echotherm ${ }^{\mathrm{TM}} \mathrm{HS}_{40}$ o heating plate from Torrey Pines Scientifics, Inc. In the following, we discuss mixtures which gave single crystals, which could be analyzed by X-ray diffraction.

For the mixture of the diboronate ester $\mathbf{E}_{2}$ and ligand L1, we were able to obtain crystals from 1,2-dichlorobenzene and 1,2,4-trichlorobenzene (Scheme 4). In both cases, linear polymers (P1 and $\left.\mathbf{P}_{2}\right)$ were formed (Figure 4). The aggregates can be described as (E2L1 $)_{2}$ macrocycles which are bridged by an additional diester $\mathbf{E} 2$ to give a polymer of formula $\left[\mathbf{E}_{2}\left(\mathbf{E}_{2} \mathbf{L} \mathbf{1}\right)_{2}\right]_{n}$. The structures of $\mathbf{P}_{1}$ and $\mathbf{P} \mathbf{2}$ are over- all very similar, with the difference being the relative orientation of adjacent $\left(\mathbf{E}_{2} \mathbf{L} \mathbf{1}\right)_{2}$ macrocycles. For both polymers, we observe $\pi-\pi$ stacking interactions between catecholate groups of adjacent polymer strands (Figures Sio and $\mathrm{S}_{11}$ ). One should note that the data quality of the structural analysis of $\mathbf{P} \mathbf{1}$ and $\mathbf{P} \mathbf{2}$ is very poor, despite the utilization of synchrotron radiation for $\mathbf{P 2}$. However, the connectivity of polymer chain could clearly be established.

Polymeric structures which consist of chains of macrocycles have - to the best of our knowledge - not been observed before for crystalline B-N adducts. The reasons for the formation of one-dimensional polymers as opposed to two-dimensional networks (Scheme $2 \mathrm{~b}$ ) are presently not clear. Possibly, small and soluble $\left(\mathbf{E}_{2} \mathbf{L} \mathbf{1}\right)_{2}$ macrocycles are first formed upon cooling. Subsequent cross-linking via additional E2 could then induce crystallization. However, it should be noted that larger macrocycles, as required for network formation, can form from very similar building blocks (see below). Therefore, we would like to propose the following alternative scenario. When a certain temperature range is reached during the cooling process, a mixture of dynamic B-N adducts is formed. Further cooling results in crystallization, the outcome of which is determined by the relative solubility of the species involved.

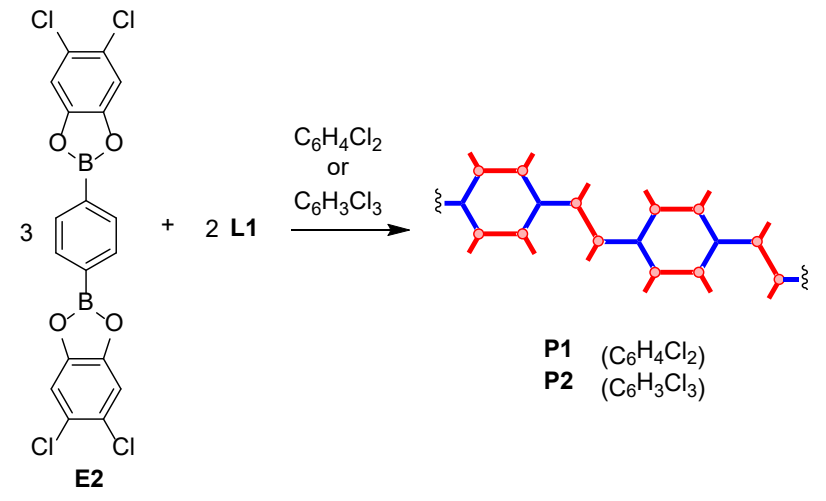

Scheme 4 Synthesis of the crystalline polymers $\mathbf{P} 1$ and $\mathbf{P} 2$.
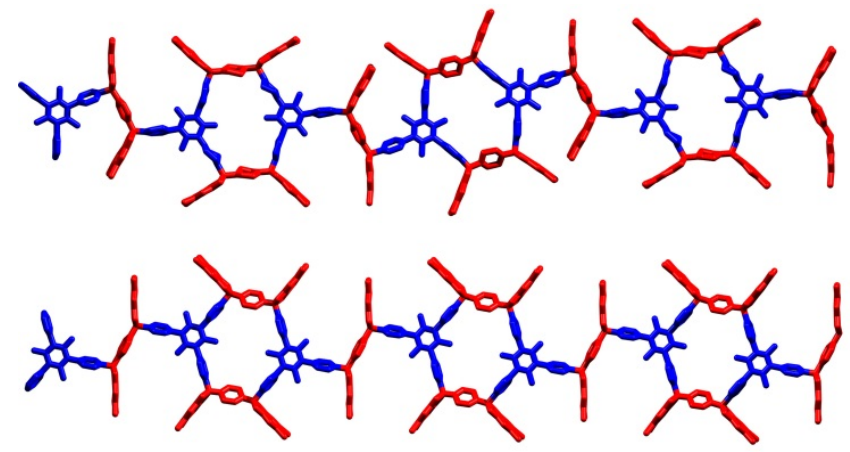

Figure 4 Parts of the molecular structures of $P_{1}$ (top) and P2 (bottom) in the crystal. Color coding: blue: L1, red: E2. Hydrogen atoms and solvent molecules have been omitted for clarity.

With the extended diboronate ester $\mathbf{E}_{3}$, we have been able to obtain two types of crystals $\left(\mathbf{P}_{\mathbf{3}}\right.$ and $\left.\mathbf{P}_{\mathbf{4}}\right)$ upon reaction with $\mathbf{L} \mathbf{1}$ in 1,2-dichlorobenzene. Both polymers have 
one-dimensional chain structures, but the composition of $\mathbf{P}_{\mathbf{3}}$ and $\mathbf{P}_{\mathbf{4}}$ is different. Whereas $\mathbf{P}_{\mathbf{3}}$ displays a structure similar to that of $\mathbf{P}_{\mathbf{1}}$ and $\mathbf{P}_{\mathbf{2}}$ (chain of macrocyles, $\left[\mathbf{E}_{\mathbf{3}}\left(\mathbf{L}_{\mathbf{1}} \mathbf{E}_{3}\right)_{2}\right]_{n}$ ), one can observe a simple $\left(\mathbf{E}_{3} \mathbf{L} \mathbf{1}\right)_{n}$ chain for polymer $\mathbf{P}_{4}$ (Scheme 5 and Figure 5). As a consequence, polymer $\mathbf{P}_{4}$ shows 'free' pyridyl groups which are not coordinated to boronate esters. Analysis of the reaction product by powder X-ray diffraction indicated that mixtures of these two polymers were always obtained, regardless of the stoichiometry of the starting materials (we have employed $\mathbf{E}_{3}$ to L1 ratios of 3:2 and 1:1).

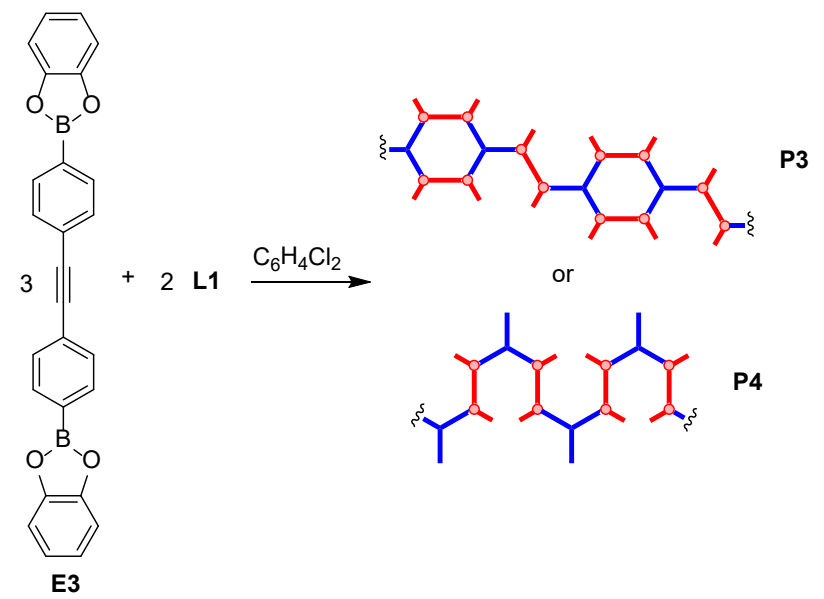

Scheme 5 Synthesis of the crystalline polymers $\mathbf{P}_{\mathbf{3}}$ and $\mathbf{P}_{\mathbf{4}}$.
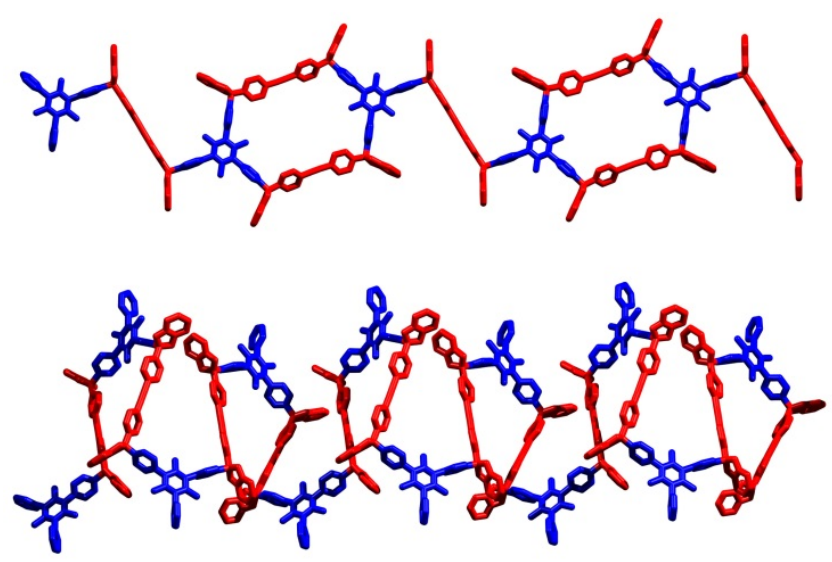

Figure 5 Parts of the molecular structures of $\mathbf{P}_{\mathbf{3}}$ (top) and $\mathbf{P}_{4}$ (bottom) in the crystal. Color coding: blue: L1, red: E3. Hydrogen atoms and solvent molecules have been omitted for clarity.

Slow cooling of a 1,2-dichlorobenzene solution of the diester $\mathbf{E}_{4}$ and the tripyridyl ligand L1 resulted in the formation of crystals containing the macrocycle Mi (Scheme 6 and Figure 6). As it was observed for polymer $\mathbf{P}_{4}$, one third of the pyridyl groups are not coordinated to boronate esters, even though a ratio of $\mathbf{E}_{4}$ to $\mathbf{L 1}_{1}$ of 3:2 was employed. The large macrocyclic structure has the composition $\left(\mathbf{E}_{4} \mathbf{L} \mathbf{1}\right)_{6}$ and a ring size of 102 atoms. It should be noted that this hexameric macrocycle is the subunit of a hypothetical two-dimensional polymeric network
(Scheme 2b). Apparently, the dative B-N bond between $\mathbf{E}_{4}$ and L1 is not sufficiently stable to promote the formation of an extended $2 \mathrm{D}$ structure with complete coordination of the pyridyl groups.

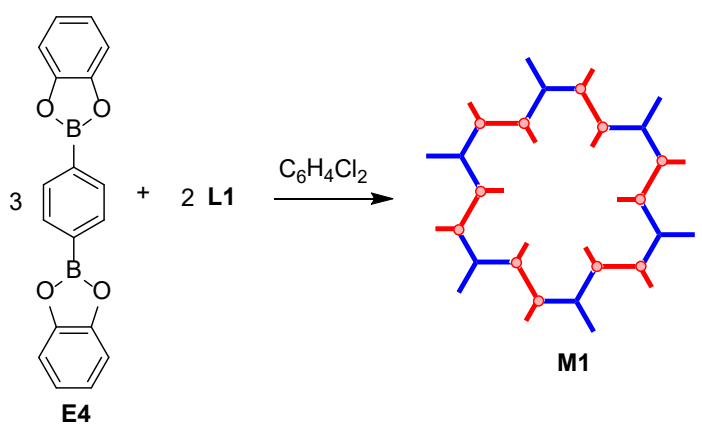

Scheme 6 Synthesis of the crystalline macrocycle M1.

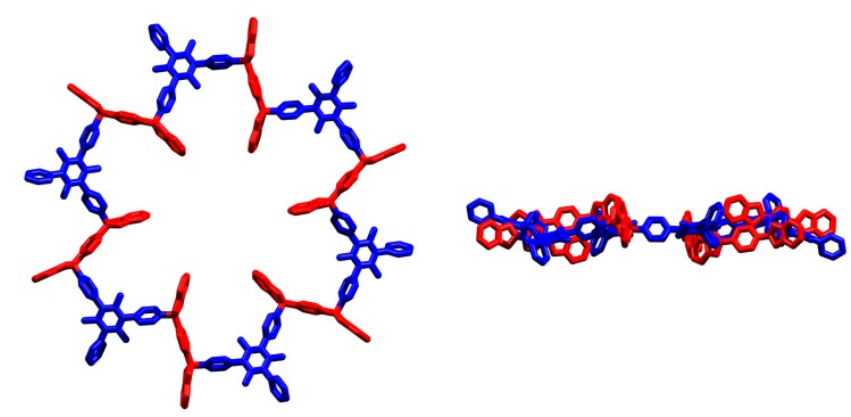

Figure 6 Molecular structures of M1 in the crystal with view from the top (left) and from the side (right). Color coding: blue: L1, red: E4. Hydrogen atoms and solvent molecules have been omitted for clarity.

For reaction mixtures containing the larger tripyridyl ligand L2, it was more difficult to obtain single crystalline material. Among several combinations which were tested, only the diboronate ester $\mathbf{E}_{\mathbf{5}}$ gave rise to a crystalline product $\left(\mathbf{P}_{\mathbf{5}}\right)$. A crystallographic analysis revealed that a onedimensional polymer with the stoichiometry $\left(\mathbf{E}_{\mathbf{5}} \mathbf{L} 2\right)_{n}$ had formed (Scheme 7 and Figure 7). Contrary to what was found for $\mathbf{P}_{\mathbf{4}}$, polymer $\mathbf{P}_{\mathbf{5}}$ features a simple zig-zag chain, similar to what has previously been observed for adducts of diboronate esters and dipyridyl ligands. ${ }^{6}$

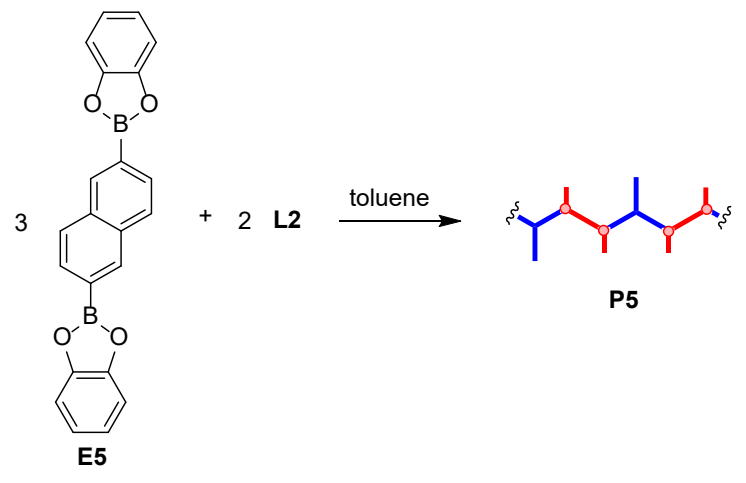

Scheme 7 Synthesis of the crystalline polymer $\mathbf{P}_{\mathbf{5}}$. 


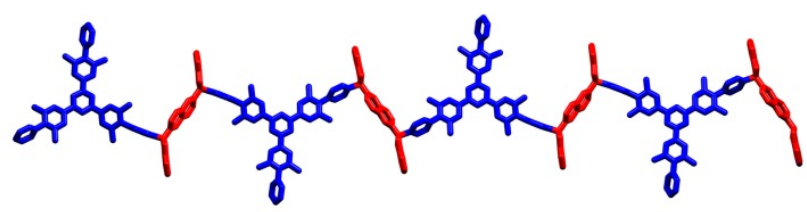

Figure 7 Part of the molecular structure of $\mathbf{P}_{\mathbf{5}}$ in the crystal. Color coding: blue: L2, red: $\mathbf{E}_{\mathbf{5}}$. Hydrogen atoms and solvent molecules have been omitted for clarity.

\section{Conclusions}

Adducts between pyridyl donor ligands and boronate esters show a preferential conformation in which the pyridine ring is nearly orthogonal to the plane defined by the three adjacent N-B-C atoms. We have exploited this preference for the crystal engineering of polymeric B-N adducts. The conformationally restricted tripyridyl ligands $\mathbf{L 1}$ and L2 were synthesized. These ligands feature pyridyl groups which are oriented perpendicular to the $\sigma_{h}$ symmetry plane of the ligand. Linear diboronate esters are expected to coordinate to the pyridyl groups in a side-on fashion, thereby promoting the formation of polymeric structures. We have examined the crystal structures of six different adducts of diboronate esters and the N-donors L1 or L2. In all cases, we observed the predicted side-on coordination of the diboronate ester. However, instead of twodimensional network structures, we observed in three cases the formation of chains of macrocycles $\left(\mathbf{P}_{\mathbf{1}}-\mathbf{P}_{\mathbf{3}}\right)$. To the best of our knowledge, this kind of structural motif has not been observed so far for crystalline B-N adducts. The strength of the dative B-N bond is not particularly high. ${ }^{9,20}$ On one hand, this characteristic is an advantage because it allows breaking the bond at higher temperatures. A heating-cooling cycle can therefore be used to induce crystallization. On the other hand, our results also demonstrate a disadvantage of using a rather weak interaction. For three crystalline adducts $\left(\mathbf{P}_{\mathbf{4}}, \mathbf{P}_{\mathbf{5}}\right.$, and $\left.\mathbf{M 1}\right)$, we observed incomplete $\mathrm{B}-\mathrm{N}$ bond formation. As a consequence, these kinds of Lewis acid-base reactions can give rise to mixtures of products and starting materials. It is expected that this problematic can be obviated to some extend by strengthening the $\mathrm{B}-\mathrm{N}$ bond via electronic effects. ${ }^{20}$ An interesting perspective for future work is the formation of three-dimensional network structures based on B-N bonds. Compounds of this kind are largely unexplored, ${ }^{14}$ and the design criteria outlined in this publication may provide a useful guideline for the preparation of such materials.

\section{ASSOCIATED CONTENT}

Supporting Information. Experimental procedures, analytical data, and crystallographic data in cif format. This material is available free of charge via the Internet at http://pubs.acs.org.

\section{AUTHOR INFORMATION}

Corresponding Author

*Email: kay.severin@epfl.ch.

\section{ACKNOWLEDGMENT}

The work was supported by the Swiss National Science Foundation and by the Ecole Polytechnique Fédérale de Lausanne (EPFL). We are grateful to the Swiss-Norwegian Beamline Consortium for providing access to synchrotron radiation.

\section{REFERENCES}

(1) Höpfl, H. J. Organomet. Chem. 1999, 581, 129-149.

(2) Icli, B.; Sheepwash, E.; Riis-Johannessen, T.; Schenk, K.; Filinchuk, Y.; Scopelliti, R.; Severin, K. Chem. Sci. 2011, 2, 1719-1721.

(3) Dhara, A.; Beuerle, F. Chem. Eur. J. 2015, 21, 17391-17396.

(4) Icli, B.; Solari, E.; Kilbas, B.; Scopelliti, R.; Severin, K. Chem. Eur. J., 2012, 18, 14867-14874.

(5) Christinat, N.; Scopelliti, R.; Severin, K. Chem. Commun., 2008, 3660-3662.

(6) Christinat, N.; Croisier, E.; Scopelliti, R.; Cascella, M.; Röthlisberger, U.; Severin, K. Eur. J. Inorg. Chem. 2oo7, 5177-5181.

(7) Sheepwash, E.; Krampl, V.; Scopelliti, R.; Sereda, O.; Neels, A.; Severin, K. Angew. Chem. Int. Ed. Engl. 2011, 50, 3034-3037.

(8) Herrera-España, A. D.; Campillo-Alvarado, G.; RománBravo, P.; Herrera-Ruiz, D.; Höpfl, H.; Morales-Rojas, H. Cryst. Growth Des. 2015, 15, 1572-1576.

(9) Luisier, N.; Scopelliti, R.; Severin, K. Soft Matter 2016, 12, 588-593.

(10) Luisier, N.; Schenk, K.; Severin, K. Chem. Commun. 2014, 50, $10233-10236$.

(11) Ito, S.; Takata, H.; Ono, K.; Iwasawa, N. Angew. Chem. Int. Ed. 2013, 52, 11045-11048.

(12) For reviews see: (a) Kubo, Y.; Nishiyabu, R.; James, T. D. Chem. Commun. 2015, 51, 2005-2020; (b) Severin, K. Dalton Trans. 2009, 5254-5264.

(13) Sheepwash, E.; Zhou, K.; Scopelliti, R.; Severin, K. Eur. J. Inorg. Chem. 2013, 2558-2563.

(14) Cruz-Huerta, J.; Salazar-Mendoza, D.; Hernández-Paredes, J.; Hernández-Ahuactzi, I. F.; Höpfl, H. Chem. Commun. 2o12, 48, 4241-4243

(15) Yuan, C.; Chang, Y.; Mao, J.; Yu, S.; Luo W.; Xu, Y.; Thayumanavan, S.; Dai, L. J. Mater. Chem. B 2015, 3, 2858-2866.

(16) Salazar-Mendoza, D.; Cruz-Huerta, J.; Höpfl, H.; Hernández-Ahuactzi, I. F.; Sanchez, M. Cryst. Growth Des. 2013, 13, 2441-2454.

(17) Li, L.; Yuan, C.; Dai, L.; Thayumanavan, S. Macromolecules 2014, 47, 5869-5876.

(18) Christinat, N.; Scopelliti, R.; Severin, K. Chem. Commun. 2004, 1158-1159.

(19) Christinat, N.; Scopelliti, R.; Severin, K. J. Org. Chem. 2oo7, 72, 2192-2200.

(20) Sheepwash, E.; Luisier, N.; Krause, M. R.; Noé, S.; Kubik, S.; Severin, K. Chem. Commun. 2012, 48, 7808-7810. 
SYNOPSIS. The combination of tripyridyl ligands and linear diboronate esters results in the formation of oligomeric or polymeric adducts featuring dative boron-nitrogen bonds. The relative orientation of the two different building blocks in the assemblies can be predicted based on a conformation analysis.

TOC graphic

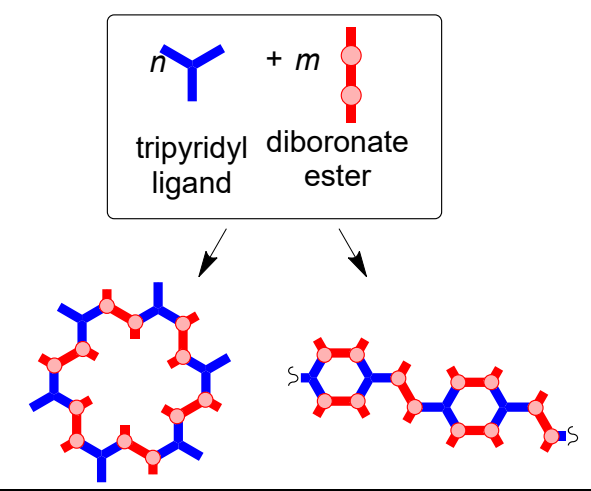

